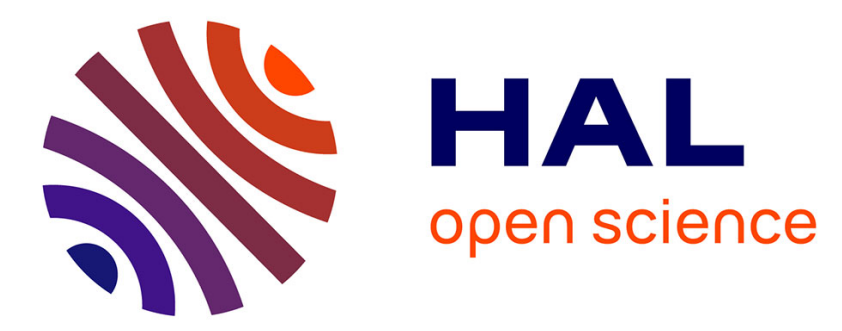

\title{
Localization of spatially distributed brain sources after a tensor-based preprocessing of interictal epileptic EEG data
}

Laurent Albera, H Becker, Ahmad Karfoul, Rémi Gribonval, A Kachenoura, S Bensaid, Lotfi Senhadji, A Hernandez, I Merlet

\section{To cite this version:}

Laurent Albera, H Becker, Ahmad Karfoul, Rémi Gribonval, A Kachenoura, et al.. Localization of spatially distributed brain sources after a tensor-based preprocessing of interictal epileptic EEG data. 2015 37th annual international conference of the IEEE Engineering in Medicine and Biology Society, Aug 2015, Milan, Italy. pp.6995 - 6998. hal-01245239

\author{
HAL Id: hal-01245239 \\ https://hal.science/hal-01245239
}

Submitted on 17 Dec 2015

HAL is a multi-disciplinary open access archive for the deposit and dissemination of scientific research documents, whether they are published or not. The documents may come from teaching and research institutions in France or abroad, or from public or private research centers.
L'archive ouverte pluridisciplinaire HAL, est destinée au dépôt et à la diffusion de documents scientifiques de niveau recherche, publiés ou non, émanant des établissements d'enseignement et de recherche français ou étrangers, des laboratoires publics ou privés. 


\title{
Localization of spatially distributed brain sources after a tensor-based preprocessing of interictal epileptic EEG data
}

\author{
L. Albera, H. Becker, A. Karfoul, R. Gribonval, A. Kachenoura, \\ S. Bensaid, L. Senhadji, A. Hernandez and I. Merlet
}

\begin{abstract}
This paper addresses the localization of spatially distributed sources from interictal epileptic electroencephalographic data after a tensor-based preprocessing. Justifying the Canonical Polyadic (CP) model of the space-time-frequency and space-time-wave-vector tensors is not an easy task when two or more extended sources have to be localized. On the other hand, the occurrence of several amplitude modulated spikes originating from the same epileptic region can be used to build a space-time-spike tensor from the EEG data. While the $C P$ model of this tensor appears more justified, the exact computation of its loading matrices can be limited by the presence of highly correlated sources or/and a strong background noise. An efficient extended source localization scheme after the tensorbased preprocessing has then to be set up. Different strategies are thus investigated and compared on realistic simulated data: the "disk algorithm" using a precomputed dictionary of circular patches, a standardized Tikhonov regularization and a fused LASSO scheme.
\end{abstract}

\section{INTRODUCTION}

ElectroEncephaloGraphy (EEG) is the noninvasive technique most indicated to capture transient events directly related to epilepsies (like interictal spikes, in particular). In the context of epilepsy surgery, the use of EEG requires to solve an electromagnetic inverse problem, that is to say to estimate the current generators underlying noisy EEG data. Theoretically, a specific electromagnetic field pattern may be generated by an infinite number of spatially distributed (extended) sources, leading to an ill-posed Brain Source Imaging (BSI) problem. In the last decades, many different regularization schemes [1] have been proposed such as the 4-ExSo-MUSIC technique [2]. However, their performance can be limited in the presence of several extended sources and background activity [3].

To overcome these problems, a tensor-based preprocessing can be applied. It consists in constructing a threeway array from the data and decomposing it using the Canonical Polyadic (CP) model [4]. Such a strategy aims at separating the different sources of interest, leading to an easier estimation of their location and spatial extent. In the past, several authors have proposed to decompose a Space-Time-Frequency (STF) tensor, which can be obtained by computing a wavelet transform [5]-[7] over the time

L. Albera, A. Karfoul, A. Kachenoura, L. Senhadji, A. Hernandez and I. Merlet are with the INSERM U1099, Rennes F-35000, France, and the Université de Rennes 1, LTSI, Rennes F-35000, France. L. Albera and R. Gribonval are with Inria, Centre Inria Rennes - Bretagne Atlantique, Rennes, F-35000, France. H. Becker is with Technicolor SA, Cesson-Sévigné F35576, France.

This work was supported by the European Research Council, PLEASE project (ERC-StG-2011-277906). dimension of the measurements. In [8], authors have considered a Space-Time-Wave-Vector (STWV) tensor, which is derived from a local spatial Fourier transform of the EEG measurements. An advantage of this approach compared to the STF analysis consists in its better robustness to correlated source activities, which is of particular interest in the context of interictal spikes when spreading of epileptic spikes is suspected between two regions.

The topic of extended source localization after the tensorbased preprocessing has been originally addressed in [8] through the Binary Coefficient Matching Pursuit (BCMP) algorithm in the context of a spherical head model and of simplistic simulated EEG signals. More recently, a more efficient method, named Disk Algorithm (DA), has been designed [9] to localize extended sources from the tensor decomposition. A detailed study of the tensor-based preprocessing methods has also been conducted [9], including an analysis of their theoretical foundation, their computational complexity, and their performance for realistic simulated data in comparison to conventional source localization algorithms such as SLORETA [10] and 4-ExSo-MUSIC.

However, justifying the CP model of the STF and STWV tensors is not an easy task when two or more extended sources have to be localized as deeply analyzed in [9]. On the other hand, the occurrence of several amplitude modulated spikes originating from the same epileptic region can be used to build a space-time-spike tensor from the EEG data [7]. While the CP model of this tensor appears more justified, the exact computation of its loading matrices can be limited by the presence of highly correlated sources or/and a strong background noise. An efficient extended source localization scheme after the tensor-based preprocessing has then to be set up. Different strategies are thus investigated and compared on realistic simulated data in terms of estimation accuracy and CPU time: DA, sLORETA and a fused LASSO scheme named SVB-SCCD [3].

\section{BSI PROBLEM FORMULATION}

The electrical activity observed at the surface of the scalp originates mostly from pyramidal cells that are arranged in the gray matter perpendicularly to the cortex. These neuronal populations are generally modeled by a grid of dipole sources located at the surface of the cortex [9, Dale and Sereno's reference]. The electric potential data, which is recorded at the $M$ electrodes of an EEG sensor array for $T$ time samples, then constitutes the superposition of all dipole signals that are transmitted to the surface of the scalp and 
some instrumentation noise $\boldsymbol{N}^{(i)}$ :

$$
\boldsymbol{X}=\boldsymbol{G}^{(e)} \boldsymbol{S}^{(e)}+\boldsymbol{G}^{(b)} \boldsymbol{S}^{(b)}+\boldsymbol{N}^{(i)}=\boldsymbol{G}^{(e)} \boldsymbol{S}^{(e)}+\boldsymbol{N}^{(i, b)}
$$

where the matrices $\boldsymbol{G}^{(e)} \in \mathbb{R}^{M \times P^{(e)}}$ and $\boldsymbol{G}^{(b)} \in \mathbb{R}^{M \times P^{(b)}}$ model the propagation of the epileptic and background dipole activities, respectively, to the scalp electrodes. Note that the global propagation matrix $\boldsymbol{G}=\left[\boldsymbol{G}^{(e)}, \boldsymbol{G}^{(b)}\right] \in \mathbb{R}^{M \times P}$ with $P=P^{(e)}+P^{(b)}$ is called the lead field matrix. It depends on spatial parameters of the head, such as the geometry of the brain, skull, and scalp as well as their conductivities. For a given head model and source space, the lead field matrix can be computed by solving numerically Poisson's equation. The matrices $\boldsymbol{S}^{(e)} \in \mathbb{R}^{P^{(e)} \times T}$ and $\boldsymbol{S}^{(b)} \in \mathbb{R}^{P^{(b)} \times T}$ contain the epileptic and background dipole signals, respectively. The matrix $\boldsymbol{N}^{(i, b)} \in \mathbb{R}^{M \times T}$ is the superposition of the background activity transmitted to the surface of the scalp and the instrumentation noise.

In the context of epilepsy, the brain sources of interest are spatially distributed and their spatial extent $\Omega^{(e)}$ can be described as the union of contiguous areas $\Omega_{r}^{(e)}(r \in$ $\{1, \ldots, R\})$ of the cortex (so-called patches) with highly correlated source activities [2]. As the activity is highly synchronized within one extended source, we can assume that all signals emitted by dipoles of the $r$-th $(r \in\{1, \ldots, R\})$ extended source are approximately equal to a signal denoted by $\boldsymbol{S}_{r,:}$ in the following. Consequently, we can approximate the data matrix by:

$$
\boldsymbol{X} \approx \sum_{r=1}^{R} \boldsymbol{A}_{:, r} \circ \boldsymbol{S}_{r,:}+\boldsymbol{N}^{(i, b)}=\boldsymbol{A} \boldsymbol{S}+\boldsymbol{N}^{(i, b)}
$$

where $\circ$ denotes the outer product operator [11]. The spatial mixing vector $\boldsymbol{A}_{:, r}$ of the epileptic patch $\Omega_{r}^{(e)}$ corresponds to the sum of the lead field vectors of all dipoles of $\Omega_{r}^{(e)}$ :

$$
\boldsymbol{A}_{:, r}=\sum_{p_{r} \in \Omega_{r}^{(e)}} \boldsymbol{G}_{:, p_{r}}=\boldsymbol{G} \boldsymbol{Z}_{:, r}
$$

where the $P$-dimensional vector $Z_{:, r}$ of ones and zeros is $P_{r}^{(e)}$-sparse with $P_{r}^{(e)}$ the number of dipoles of the epileptic patch $\Omega_{r}^{(e)}$. The BSI problem consists then in recovering the coefficient matrix $\boldsymbol{Z} \in \mathbb{R}^{P \times R}$ (3) from the EEG data $\boldsymbol{X}$ (2) using the known lead field matrix $\boldsymbol{G}$.

\section{TENSOR-BASED BSI SOLUTIONS}

The analysis of space-time EEG signals using the CP decomposition of a specific third order tensor has been originally proposed in [12]. More precisely, the author canonically decomposed a space-time-subject tensor built from the data by considering that there were observations of the same phenomenon on several subjects. If such a consideration is valid for the analysis of event-related potentials, it does not hold in the processing of epileptic EEG signals since the latter are patient specific. Nevertheless, as described in [7] and briefly recalled hereafter some information redundancies can be used in interictal epileptic EEG data allowing us to build a Space-Time-Spike (STS) tensor. Three different approaches, namely DA, sLORETA and SVB-SCCD, are then described hereafter in order to derive the location and the spatial extent of the epileptic regions from the $\mathrm{CP}$ decomposition of the considered tensor.

\section{A. Tensor-based preprocessing}

Spikes related to the same brain region appear several times in the interictal epileptic EEG data. The latter can then be segmented in time slots corresponding to different spikes. The $J$ different space-time events of size $(M \times K)$ can thus be mutually synchronized with respect to the top of each spike and concatenated in a STS tensor $\mathcal{X} \in \mathbb{R}^{M \times K \times J}$ enjoying the following low-rank $\mathrm{CP}$ model:

$$
\mathcal{X}=\sum_{r=1}^{R} \boldsymbol{A}_{:, r} \circ \boldsymbol{B}_{:, r} \circ \boldsymbol{C}_{:, r}+\mathcal{N}
$$

where each $\boldsymbol{A}_{:, r} \circ \boldsymbol{B}_{:, r} \circ \boldsymbol{C}_{:, r}$ is a rank-1 three-way array and where the third order array $\mathcal{N}$ represents the model residual. More particularly, $\boldsymbol{A}_{:, r}$ and $\boldsymbol{B}_{:, r}$ denote the spatial mixing vector and the spike-like pattern, respectively, of the $r$-th epileptic extended source. The weights of the length- $K$ spike-like pattern $\boldsymbol{B}_{:, r}$ characterizing the spikes of the $r$-th source are given by the $J$ elements of $C_{:, r}$. Sometimes we use the notation $\mathcal{X}(\boldsymbol{A}, \boldsymbol{B}, \boldsymbol{C})$ to refer to the third order tensor $\mathcal{X}$ with its loading matrices $\boldsymbol{A}=$ $\left[\boldsymbol{A}_{:, 1}, \cdots, \boldsymbol{A}_{:, R}\right] \in \mathbb{R}^{M \times R}, \boldsymbol{B}=\left[\boldsymbol{B}_{:, 1}, \cdots, \boldsymbol{B}_{:, R}\right] \in \mathbb{R}^{K \times R}$ and $\boldsymbol{C}=\left[\boldsymbol{C}_{:, 1}, \cdots, \boldsymbol{C}_{:, R}\right] \in \mathbb{R}^{J \times R}$.

The CP model (4) defines a rank- $R$ approximation of tensor $\mathcal{X}$. If $R=\operatorname{rank}(\mathcal{X})$ then the model residual is null and the CP decomposition is exact. In the latter case, there is almost surely a finite number of decompositions of the tensor $\mathcal{X}$ into the three loading matrices $\boldsymbol{A}, \boldsymbol{B}$ and $C$, up to scale and permutation indeterminacies, if $R<$ $(M K J) /(M+K+J-2)$ [11]; tighter bounds also give sufficient conditions for uniqueness such as the condition based on Kruskal rank [11]. Note that Alternating Least Squares (ALS) algorithm [11] was used to compute the CP decomposition of $\mathcal{X}$.

\section{B. Extended source localization}

The objective of extended source localization consists in recovering the coefficient matrix $Z$ (3). This can be achieved from an estimate $\widehat{\boldsymbol{A}}=\left[\widehat{\boldsymbol{A}}_{:, 1}, \ldots, \widehat{\boldsymbol{A}}_{:, R}\right]$ of the spatial mixing matrix $\boldsymbol{A}$ computed by means of the CP decomposition of $\mathcal{X}$ (4), which permits to identify the grid dipoles for each patch separately. In principle, any localization algorithm such as the fused LASSO approach SVB-SCCD [3] and SLORETA [10] that act on a vector of spatial measurements can be employed to this end. Here, we briefly describe DA [9], a surface grid based version of SLORETA and SVBSCCD.

1) $D A$ : The concept underlying DA consists in recovering the extended source from a number of small circular-shaped patches of grid dipoles, the disks. Each extended source is then obtained by the union of several disks. For each grid dipole, several disks composed of the 0 to $D_{\max }-1$ 
nearest dipoles and the current grid dipole as central point are determined. Assuming equal coefficients for all grid dipoles belonging to the extended source, the spatial mixing vector of each disk is computed by adding the lead field vectors of all grid dipoles belonging to the disk. The reconstructed spatial mixing vector of the $j$-th disk $\left(j \in\left\{1, \ldots, D_{\max } P\right\}\right)$ is given by $\boldsymbol{G} \widehat{\boldsymbol{u}}_{j}$ where the elements of the coefficient vector $\widehat{\boldsymbol{u}}_{j}$ that are associated with dipoles belonging to the disk are equal to 1 whereas all other elements are 0 . To determine which disks of the parameter space best describe the $r$-th patch, the spatial mixing vectors $\widehat{\boldsymbol{u}}_{j}$ of all disks are then compared to the estimated spatial mixing vector $\widehat{\boldsymbol{A}}_{:, r}$ of the $r$-th source by means of the squared normalized inner product:

$$
\varphi_{1}(\boldsymbol{u})=\left(\widehat{\boldsymbol{A}}_{:, r}^{\top} \boldsymbol{G} \boldsymbol{u}\right)^{2}\left(\widehat{\boldsymbol{A}}_{:, r}^{\top} \widehat{\boldsymbol{A}}_{:, r} \boldsymbol{u}^{\top} \boldsymbol{G}^{\top} \boldsymbol{G} \boldsymbol{u}\right)^{-1}
$$

The $r$-th patch is then estimated as the union of a certain number of disks which are associated with the highest values of the metric $\varphi_{1}(5)$.

2) sLORETA: sLORETA as all minimum norm solutions is based on the assumption that among all possible solutions that explain the data, the one with minimal energy is the most likely. In this context, the idea of the conventional sLORETA algorithm consists in standardizing the minimum norm solution $\widehat{\boldsymbol{Z}}_{:, r}$ by weighting its components by their estimated variances. Thanks to an inversion lemma, the minimum argument of the following objective function:

$$
\varphi_{2}(\boldsymbol{u})=\left\|\widehat{\boldsymbol{A}}_{:, r}-\boldsymbol{G} \boldsymbol{u}\right\|^{2}+\lambda\|\boldsymbol{u}\|^{2}
$$

where $\lambda$ is a regularization parameter, can be written as $\widehat{\boldsymbol{u}}=\boldsymbol{Q} \widehat{\boldsymbol{A}}_{:, r}$ with $\boldsymbol{Q}=\boldsymbol{G}^{\top}\left(\boldsymbol{G} \boldsymbol{G}^{\top}+\lambda \mathbf{I}_{M}\right)^{-1} \in \mathbb{R}^{P \times M}$ the Tikhonov-regularized inverse matrix of $\boldsymbol{G}$ and where $\mathbf{I}_{M}$ denotes the identity matrix of $R^{M \times M}$. The idea of sLORETA consists in weighting solution $\widehat{\boldsymbol{u}}$ by the inverse of the diagonal matrix $(\operatorname{diag}\{\operatorname{diag}\{\boldsymbol{F}\}\})^{1 / 2}$ where the matrix $\boldsymbol{F}$ of the form:

$$
\boldsymbol{F}=\boldsymbol{G}^{\top}\left(\boldsymbol{G} \boldsymbol{G}^{\top}+\lambda \mathbf{I}_{M}\right)^{-1} \boldsymbol{G}
$$

is the covariance matrix of $\widehat{\boldsymbol{u}}$. The $p$-th coefficient $(p \in$ $\{1, \ldots, P\})$ of $\widehat{\boldsymbol{Z}}_{:, r}$ is then computed as:

$$
\widehat{\boldsymbol{Z}}_{p, r}=\left(F_{p, p}\right)^{-\frac{1}{2}} \boldsymbol{Q}_{p,:} \widehat{\boldsymbol{A}}_{:, r}
$$

The resulting coefficient vector $\widehat{\boldsymbol{Z}}_{:, r}$ contains components with continuous values. The $r$-th patch is estimated by identifying the components of $\widehat{\boldsymbol{Z}}_{:, r}$ which exceed a certain threshold.

3) SVB-SCCD: The SVB-SCCD algorithm [3] computes a sparse piece-wise constant spatial solution, by imposing sparsity on both the coefficient vector $Z_{:, r}$ and its variational map. The latter can be computed by applying a linear transform, characterized by the matrix $\boldsymbol{V}$, to the coefficient vector, which is equivalent to computing the total variation on the discretized cortical surface. The components $V_{\ell, p}$ of $\boldsymbol{V} \in \mathbb{R}^{L \times P}$ where $L$ is the number of edges of the triangular grid, are given by:

$$
V_{\ell, p}=\left\{\begin{array}{cl}
1 & \text { if } p=p_{\ell, 1} \\
-1 & \text { if } p=p_{\ell, 2} \\
0 & \text { otherwise }
\end{array}\right.
$$

where $p_{\ell, 1}$ and $p_{\ell, 2}$ are the indices of the dipoles sharing the $\ell$-th edge. This leads to the following cost function:

$$
\varphi_{3}(\boldsymbol{u})=\frac{1}{2}\left\|\widehat{\boldsymbol{A}}_{:, r}-\boldsymbol{G u}\right\|_{\mathrm{F}}^{2}+\lambda\left(\|\boldsymbol{V} \boldsymbol{u}\|_{1}+\alpha\|\boldsymbol{u}\|_{1}\right)
$$

whose minimization giving the coefficient vector $\widehat{Z}_{:, r}$ is equivalent to the sparse TV (or fused LASSO) approach. The latter permits us to adjust the size of the reconstructed source regions by varying the new regularization parameter $\alpha$. In addition, it prevents the estimated signal vector from being amplitude-biased, which is a problem that frequently arises using only the TV norm. Setting $\alpha=1$ leads to very focal source estimates, whereas small $\alpha$ only avoid the amplitude bias, but do not influence the size of the reconstructed source regions. The regularization parameter $\lambda$ regulates the impact of the source priors. Note that the Alternating Direction Method of Multipliers (ADMM) [3, Boyd et al.'s reference] was used to minimize $\varphi_{3}(10)$.

\section{Simulations}

In this section, we compare the performance of STS-DA, STS-SCCD and STS-sLORETA (i.e. the three tensor-based BSI methods described in section III) on realistic simulated interictal epileptic EEG data.

\section{A. General settings and performance criteria}

For STS-SCCD, the regularization parameter $\lambda$ was adjusted such that the reconstruction error lies within a confidence interval of 95 to $99 \%$ of the noise power and we used a fixed parameter $\alpha=0.67$ because we found that this leads to reasonable results for the considered scenario.

The EEG signals were generated for $N=91$ electrodes using a realistic head model with three compartments that represent the brain, the skull, and the scalp. The source space consists of $D=19626$ dipoles corresponding to the triangles of the cortical surface mesh with orientations perpendicular to the cortical surface. A BEM method ${ }^{1}$ was used to compute the lead field matrix.

We considered three patches, located in the inferior frontal gyrus (patch InfFr), the occipital temporal junction (patch OccTe), and the mid temporal gyrus (patch MidTe). Each one is composed of 100 adjacent dipoles. The patches InfFr and OccTe were attributed different epileptic spike signals comprising 200 time samples (at $256 \mathrm{~Hz}$ sampling frequency) that were segmented from stereotactic EEG (SEEG) recordings of a patient suffering from epilepsy. Then we generated 40 different realizations for each of these two signals, one for each patch dipole, by introducing small variations in amplitude and delay. Assuming that the patch MidTe was activated due to a propagation of the epileptic activity of the patch OccTe, we used the same signals for the dipoles of these two patches, but introduced a delay of $8 \mathrm{~ms}$ due to the distance between the patches.

All source dipoles that do not belong to a patch were attributed Gaussian background activity with an amplitude that was adjusted to the amplitude of the SEEG signals

${ }^{1}$ ASA, ANT, Enschede, Netherlands 


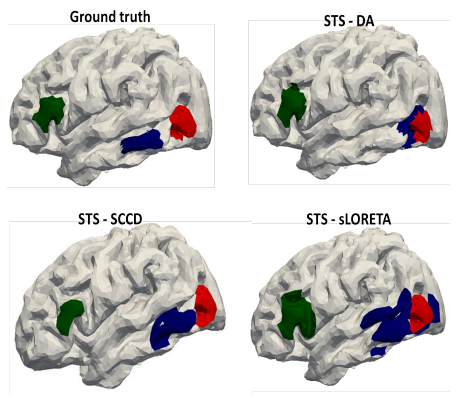

Fig. 1. BSI results obtained with the three tested algorithms.

between epileptic spikes, thus leading to a realistic Signal to Noise Ratio (SNR) such that $\left\|\boldsymbol{G}^{(e)} \boldsymbol{S}^{(e)}\right\|_{\mathrm{F}}^{2} /\left\|\boldsymbol{N}^{(i, b)}\right\|_{\mathrm{F}}^{2} \approx 1$.

The performance of the three BSI tensor-based methods was assessed computing both the CPU time and the Dipole Localization Error (DLE). If $\Omega^{(e)}$ and $\widehat{\Omega}^{(e)}$ denote the original and estimated sets of indices of all epileptic dipoles, $P^{(e)}$ and $\hat{P}^{(e)}$ are the numbers of original and estimated epileptic dipoles, and $\boldsymbol{r}_{p}$ denotes the position of the $p$-th epileptic dipole, then the DLE is defined by:

$$
\begin{aligned}
\mathrm{DLE}=\frac{1}{2 P^{(e)}} \sum_{p \in \Omega^{(e)}} \min _{\hat{p} \in \widehat{\Omega}^{(e)}}\left\|\boldsymbol{r}_{p}-\boldsymbol{r}_{\hat{p}}\right\|+ \\
\frac{1}{2 \hat{P}^{(e)}} \sum_{\hat{p} \in \widehat{\Omega}^{(e)}} \min _{p \in \Omega^{(e)}}\left\|\boldsymbol{r}_{p}-\boldsymbol{r}_{\hat{p}}\right\|
\end{aligned}
$$

\section{B. Computer results}

The performance achieved with the three BSI tensor-based algorithms in terms of DLE and CPU time averaged over 40 realizations is summarized in table I. The DLE values

\begin{tabular}{|c|c|c|}
\hline methods & DLE in cm & CPU time in s \\
\hline STS-DA & 3.12 & 409 \\
STS-SCCD & 0.8991 & 282 \\
STS-sLORETA & 6.9 & 112 \\
\hline
\end{tabular}

TABLE I

PERFORMANCE OF TENSOR-BASED BSI ALGORITHMS.

show that the STS-SCCD algorithm clearly gives the best compromise between estimation accuracy and processing time for the considered multi-patch scenario. Recall that the latter involves two highly correlated patches and one decorrelated patch. Consequently, two columns of the loading matrix $\boldsymbol{B}$ are quasi-collinear and the ALS-like rank$R$ CP decomposition of $\mathcal{X}$ does not succeed in perfectly estimating the spatial mixing matrix $\boldsymbol{A}$. In fact, one column of $\boldsymbol{A}$ corresponding to the spatial mixing vector of patch InfFr appears to be well estimated contrarily to the two other columns of $\boldsymbol{A}$. In other words, the tensor-based preprocessing allows us to extract only one patch among the three patches. Note that it is a good issue provided that the two nonseparated sources can be also localized from the estimated mixing matrix $\widehat{\boldsymbol{A}}$. The good behavior of STS-SCCD can also be seen in figure 1, where we illustrate an example of the BSI results obtained with the different methods. Contrarily to
STS-SCCD, STS-sLORETA gives a blurred solution while STS-DA finds only two patches.

\section{CONCLUSION}

This paper addresses the localization of spatially distributed sources from interictal epileptic electroencephalographic data after the canonical polyadic decomposition of a space-time-spike tensor built from the data. Different strategies of extended source localization are investigated, namely the "disk algorithm" using a precomputed dictionary of circular patches, a standardized Tikhonov regularization and a fused LASSO scheme. The best compromise between estimation accuracy and processing time is offered by the fused LASSO approach. Future work will include i) the use of other tensor decompositions such as the block term decomposition [13], to deal with the case of extended sources and ii) the design of constrained tensor decomposition algorithms (i.e. the structure of the spatial mixing matrix is imposed during the tensor decomposition step).

\section{REFERENCES}

[1] H. BECKER, L. ALBERA, P. COMON, R. GRIBONVAL, F. WENDLING, and I. MERLET, "A performance study of various brain source imaging approaches," in ICASSP 14, 2014 IEEE International Conference on Acoustics Speech and Signal Processing, Florence, Italy, May 4-9 2014.

[2] G. BIROT, L. ALBERA, F. WENDLING, and I. MERLET, "Localisation of extended brain sources from EEG/MEG: the exso-music approach," NeuroImage, vol. 56, no. 1, pp. 102-113, May 2011.

[3] H. BECKER, L. ALBERA, P. COMON, R. GRIBONVAL, and I. MERLET, "Fast, variation-based methods for the analysis of extended brain sources," in EUSIPCO 14, XXII European Signal Processing Conference, Lisbon, Portugal, September 1-5 2014, pp. $41-45$.

[4] F. L. HITCHCOCK, "Multiple invariants and generalized rank of a p-way matrix or tensor," J. Math. and Phys., vol. 7, no. 1, pp. 39-79, 1927.

[5] F. MIWAKEICHI, P. A. VALDES-SOSA E. MARTINEZ-MONTES, N. NISHIYAMA, H. MIZUHARA, and Y. YAMAGUCHI, "Decomposing EEG data into space-time-frequency components using parallel factor analysis," NeuroImage, vol. 22, pp. 1035-1045, 2004.

[6] E. ACAR, C. AYKUT-BINGOL, H. BINGOL, R. BRO, and B. YENER, "Multiway analysis of epilepsy tensors," Bioinformatics, vol. 23, no. 13, pp. i10-i18, 2007.

[7] W. DEBURCHGRAEVE, P. J. CHERIAN, M. DE VOS, R. M SWARTE, J. H. BLOK, G. H. VISSER, P. GOVAERT, and S. VAN HUFFEL, "Neonatal seizure localization using ParaFac decomposition," Clinical Neurophysiology, vol. 120, pp. 1787-1796, 2009.

[8] H. BECKER, P. COMON, L. ALBERA, M. HAARDT, and I. MERLET, "Multi-way space-time-wave-vector analysis for eeg source separation," Signal Processing, vol. 92, no. 4, pp. 1021-1031, April 2012.

[9] H. BECKER, L. ALBERA, P. COMON, M. HAARDT, G. BIROT, F. WENDLING, M. GAVARET, C. G. BENAR, and I. MERLET, "EEG extended source localization: tensor-based vs. conventional methods," Elsevier NeuroImage, vol. 96, pp. 143-157, August 2014.

[10] R. D. PASCUAL-MARQUI, "Standardized low resolution brain electromagnetic tomography (sLORETA): technical details," Methods and Findings in Experimental and Clinical Pharmacology, 2002.

[11] P. COMON, X. LUCIANI, and A. L. F. DE ALMEIDA, "Tensor decompositions, alternating least squares and other tales," Journal of Chemometrics, vol. 23, April 2009.

[12] J. MOCKS, "Topographic components model for event-related potentials and some biophysical considerations," IEEE Transactions on Biomedical Engineering, vol. 35, no. 6, pp. 482-484, June 1988.

[13] B. HUNYADI, D. CAMPS, L. SORBER, W. VAN PAESSCHEN, M. DE VOS, S. VAN HUFFEL, and L. DE LATHAUWER, "Block term decomposition for modelling epileptic seizures," EURASIP Journal on Advances in Signal Processing. 